\title{
The Applicability of the International Staging System in Chinese Patients with Multiple Myeloma Receiving Bortezomib or Thalidomide-Based Regimens as Induction Therapy: A Multicenter Analysis
}

\author{
Jing Lu, ${ }^{1}$ Jin Lu, ${ }^{2}$ Aijun Liu, ${ }^{3}$ Weijun Fu, ${ }^{1}$ Juan Du, ${ }^{1}$ Xiaojun Huang, ${ }^{2}$ \\ Wenming Chen, ${ }^{3}$ and Jian Hou ${ }^{1}$ \\ ${ }^{1}$ Department of Hematology, The Myeloma and Lymphoma Center, Changzheng Hospital, The Second Military Medical University, \\ 415 Fengyang Road, Shanghai 200003, China \\ ${ }^{2}$ Peking University People's Hospital and Peking University Institute of Hematology, No. 11 Xizhimen South Street, \\ Beijing 100044, China \\ ${ }^{3}$ Department of Hematology, Beijing Chaoyang Hospital, Capital Medical University, 8 Workers Stadium South Road, \\ Beijing 100020, China
}

Correspondence should be addressed to Xiaojun Huang; xjhrm@medmail.com.cn, Wenming Chen; 13910107759@163.com, and Jian Hou; houjian167@sohu.com

Received 23 July 2015; Accepted 13 September 2015

Academic Editor: Abdel Kareem Azab

Copyright ( 92015 Jing Lu et al. This is an open access article distributed under the Creative Commons Attribution License, which permits unrestricted use, distribution, and reproduction in any medium, provided the original work is properly cited.

\begin{abstract}
The International Staging System (ISS) is the most important prognostic system for multiple myeloma (MM). It was identified in the era of conventional agents. The outcome of MM has significantly changed by novel agents. Thus the applicability of ISS system in the era of novel agents in Chinese patients needs to be demonstrated. We retrospectively analyzed the clinical outcomes and prognostic significance of ISS system in 1016 patients with newly diagnosed multiple myeloma in Chinese patients between 2008 and 2012, who received bortezomib- or thalidomide-based regimens as first-line therapy. The median overall survival (OS) of patients for ISS stages I/II/III was not reached/55.4 months/41.7 months ( $p<0.001)$, and the median progression-free survival (PFS) was 30/29.5/25 months ( $p=0.072$ ), respectively. Statistically significant difference in survival was confirmed among three ISS stages in thalidomide-based group, but not between ISS stages I and II in bortezomib-based group. These findings suggest that ISS system can predict the survival in the era of novel agents in Chinese MM patients, and bortezomib may have the potential to partially overcome adverse effect of risk factors on survival, especially in higher stage of ISS system.
\end{abstract}

\section{Introduction}

Multiple myeloma (MM) is the second most common hematological malignancy, accounting for $10 \%$ of all neoplastic hematologic disorders. It is characterized by significant heterogeneities in clinical manifestations and prognosis. The median overall survival (OS) for MM is about 4-5 years, but the OS is highly variable in different MM patients; some patients with aggressive disease courses may die in a few months after diagnosis, while other patients with indolent courses may live more than ten years [1]. Consequently, it is important to find a simple and robust stratification system to predict prognosis and help to optimize treatment strategy early in the course of myeloma [2].

Exploring a useful prognostic system has been a topic of interest in the myeloma filed since the past forty years, and considerable progresses have been made now. The DurieSalmon system was established in 2005 and worldwide used since then. This system can divide patients into three stages by tumor burden [3]. However, this system is complicated and not objective. In 2005 the International Myeloma Working Group (IMWG) developed a new stage system called the 
TABLE 1: The definition of the ISS system.

\begin{tabular}{lcc}
\hline ISS stage & \multicolumn{1}{c}{ Definition } & Median OS \\
\hline Stage I & $\begin{array}{c}\mathrm{S} \beta_{2} \mathrm{M} \text { less than } 3.5 \mathrm{mg} / \mathrm{L} \text { plus } \\
\text { serum albumin } \geq 3.5 \mathrm{~g} / \mathrm{dL}\end{array}$ & 64 months \\
Stage II & $\begin{array}{c}\text { Neither stage I nor stage III } \\
\text { Stage III }\end{array}$ & 44 months \\
\hline
\end{tabular}

ISS: International Staging System, OS: overall survival.

$\mathrm{S} \beta_{2} \mathrm{M}$ : serum beta 2 microglobulin.

International Staging System (ISS), which was relying on the combinations of two easily available and objective prognostic variables (serum beta 2 microglobulin $\left(\mathrm{S} \beta_{2} \mathrm{M}\right)$ and serum albumin): ISS stage I, S $\beta_{2}$ M less than $3.5 \mathrm{mg} / \mathrm{L}$ plus serum albumin $\geq 3.5 \mathrm{~g} / \mathrm{dL}$; ISS stage II, neither stage I nor stage III; and ISS stage III, $\mathrm{S} \beta_{2} \mathrm{M} \geq 5.5 \mathrm{mg} / \mathrm{L}$ [4] (Table 1). The ISS system is the most important and commonly used stage system today. However, difficulties have been encountered now. When ISS system was defined, it contained data from patient with MM between 1981 and 2002. All of these patients were treated by conventional agents, not exposed to novel agents. However current treatment strategies for MM have been completely changed during the last decade by the introduction of novel agents. Novel agents such as bortezomib and thalidomide have become the most important component in MM therapy and dramatically improved the response rate, progression-free survival (PFS), and even OS of MM patients [5]. Thus the prognostic value of ISS system in the era of novel agents is still in debate.

Although recent studies had been focused on the applicability of ISS in the era of novel agents, until recently there was no conclusion. One study indicated that the ISS system was still robust after introduction of thalidomide in Greece [6]. A study from Dimopoulos et al. then demonstrated the applicability of ISS in MM patients with renal dysfunction [7]. Another study from Yang et al. indicates that ISS was not suitable for patients who underwent hematopoietic stem cell transplantation (HSCT) after the introduction of thalidomide [8]. Novel agents are well tolerated and have been recommended as the first-line choice at induction chemotherapy in National Comprehensive Cancer Network (NCCN), European Society for Medical Oncology (ESMO) guideline $[9,10]$. The majority of patients received at least one kind of novel agents, such as bortezomib, thalidomide, or lenalidomide at induction therapy now. However, data is still less accurate for the prognostic significance of ISS in those who were acutely treated with novel agents at induction therapy in Chinese patients.

Therefore, we investigated a consecutive cohort of patients with MM who were treated with bortezomib- or thalidomide-based regimens as induction treatment in three Chinese centers, to validate the prognostic significance of the ISS in the era of novel agents in Chinese patients.

\section{Methods}

2.1. Patients and Treatment. A total of 1016 newly diagnosis symptomatic MM patients were enrolled between August
2006 and December 2012, from three Chinese myeloma centers (Department of Hematology at Changzheng Hospital, Peking University People's Hospital and Chaoyang Hospital). All of patients were diagnosed according to IMWG criteria [11]. The approvals were obtained from the Scientific Committee of three hospitals for the use of patients' medical records and publication of these data.

Patients who received at least two courses of one novel agent based therapy in the first-line treatment were included in this study. Thalidomide and bortezomib were introduced in the treatment of MM patients in China in 1999 and 2005, respectively, while lenalidomide was not available until 2013, before this analysis was conducted. The patients were divided into two groups by the type of the first-line regimens, bortezomib-based group and thalidomide-based group.

Patients' characteristics at diagnosis including gender, age, immunophenotype, ISS stage, peripheral neuropathy $(\mathrm{PN})$, hemoglobin, platelets, bone marrow (BM) plasma cell percentage, serum calcium, serum albumin, $S \beta_{2} M$, serum lactate dehydrogenase (LDH), and serum creatinine were collected. Table 2 reported details regarding patients' clinical and hematological features.

2.2. Follow-Up. The last follow-up was conducted in March 2013. The primary end point for this study was OS, while secondary end points were PFS and response rate. OS was defined as the time between the diagnosis and death of any cause or until the last follow-up. PFS was defined as the time between the diagnosis and progression or until the last follow-up. Response rate to induction therapy was defined according to IMWG criteria. Patients were considered responsive when they achieved at least partial response (PR) in the first-line treatment.

2.3. Statistical Analysis. Statistical analyses were performed using SPSS version 18.0. Survival curves were plotted by using the Kaplan-Meier method. OS between the stages were tested using the log-rank test, with $p<0.05$ taken as level of significance.

\section{Results}

3.1. Patient Characteristics. As showed in Table 2, 1016 patients with MM were enrolled in this study, 60.5\% were male, the median age was 59 years, and the major subtypes were $\operatorname{IgG}(44.1 \%)$, IgA (22.2\%), and light chain $(23.1 \%)$. At diagnosis, $61.5 \%$ of patients had anemia (defined as hemoglobin $<10 \mathrm{~g} / \mathrm{dL}$ ), $19.2 \%$ had renal dysfunction (defined as serum creatinine $(\mathrm{Cr}) \geq 2 \mathrm{mg} / \mathrm{mL}$ ), and $36.3 \%$ of patients' bone marrow plasma cell in filtration was more than $40 \%$.

We divided 1016 patients into two groups by the types of novel agents in the first-line treatment, 709 patients in bortezomib-based group (defined as at least received 2 cycles of bortezomib-based treatment in first-line treatment) and 307 patients in thalidomide-based group (defined as at least received 2 cycles of thalidomide-based treatment in first-line treatment). The regimens of first-line therapy in each group were also listed in Table 2. The number of patients exposed to both bortezomib and thalidomide in our database is too 
TAble 2: Patient characteristics at diagnosis according to the type of novel agents in the first-line therapy.

\begin{tabular}{|c|c|c|c|c|}
\hline Parameters & Total (\%) & Bortezomib-based group (\%) & Thalidomide-based group (\%) & $p$ value \\
\hline Patient $(n)$ & 1016 & 709 & 307 & \\
\hline Male & $59.7 \%$ & $60.1 \%$ & $59.0 \%$ & 0.394 \\
\hline Age $>60$ years & $44.2 \%$ & $40.3 \%$ & $53.1 \%$ & 0.0001 \\
\hline Hemoglobin $<10$ g/dL & $61.5 \%$ & $60.2 \%$ & $64.8 \%$ & 0.253 \\
\hline Creatinine $\geq 2 \mathrm{mg} / \mathrm{mL}$ & $19.2 \%$ & $20.3 \%$ & $16.4 \%$ & 0.183 \\
\hline BM plasma cell percentage $\geq 40 \%$ & $36.3 \%$ & $38.8 \%$ & $30.2 \%$ & 0.012 \\
\hline Platelet counts $<130 * 10^{9} / \mathrm{L}$ & $30.9 \%$ & $31.7 \%$ & $29.2 \%$ & 0.454 \\
\hline $\mathrm{PN}$ & $12.3 \%$ & $10.7 \%$ & $15.4 \%$ & 0.063 \\
\hline $\mathrm{LDH} \geq 245 \mathrm{U} / \mathrm{L}$ & $14.6 \%$ & $14.9 \%$ & $13.8 \%$ & 0.689 \\
\hline Albumin $(<35 \mathrm{~g} / \mathrm{L})$ & $47.4 \%$ & $43.6 \%$ & $56.1 \%$ & 0.0001 \\
\hline$\beta_{2}-\mathrm{MG}(\geq 3.5 \mathrm{mg} / \mathrm{L})$ & $55.3 \%$ & $56.1 \%$ & $53.4 \%$ & 0.028 \\
\hline DS stage & & & & 0.483 \\
\hline I & $2.5 \%$ & $2.2 \%$ & $3.6 \%$ & \\
\hline II & $9.5 \%$ & $9.3 \%$ & $10.1 \%$ & \\
\hline III & $88.0 \%$ & $88.5 \%$ & $86.3 \%$ & \\
\hline ISS stage & & & & 0.343 \\
\hline I & $22.7 \%$ & $24.0 \%$ & $26.4 \%$ & \\
\hline II & $33.6 \%$ & $33.3 \%$ & $34.2 \%$ & \\
\hline III & $43.7 \%$ & $42.7 \%$ & $45.9 \%$ & \\
\hline Myeloma type & & & & 0.050 \\
\hline $\operatorname{IgG}$ & $44.1 \%$ & $42.6 \%$ & $47.6 \%$ & \\
\hline $\operatorname{Ig} \mathrm{A}$ & $22.2 \%$ & $23.6 \%$ & $19.2 \%$ & \\
\hline $\operatorname{IgD}$ & $7.4 \%$ & $8.5 \%$ & $4.9 \%$ & \\
\hline$\kappa$ light chain & $12.0 \%$ & $12.1 \%$ & $11.7 \%$ & \\
\hline$\lambda$ light chain & $11.1 \%$ & $11.0 \%$ & $11.4 \%$ & \\
\hline others & $3.2 \%$ & $2.2 \%$ & $5.2 \%$ & \\
\hline Regimens of the first-line therapy & & $\mathrm{PAD} / \mathrm{VD} / \mathrm{BCD} / \mathrm{V}-\mathrm{DECP}$ & TAD/TD/MPT/CTP/T-DECP & \\
\hline$\geq \mathrm{PR}$ to the first-line therapy & $80 \%$ & $84.1 \%$ & $68.8 \%$ & 0.0001 \\
\hline
\end{tabular}

BM: bone marrow, PN: peripheral neuropathy, LDH: lactate dehydrogenase, DS stage: Durie-Salmon stage, ISS: International Staging System, OS: overall survival, PAD: Bortezomib (Velcade), Adriamycin, and Dexamethasone, VD: Bortezomib (Velcade) and Dexamethasone, BCD: Bortezomib (Velcade), Cyclophosphamide, and Dexamethasone, V-DECP: Bortezomib Cisplatin, Etoposide, Cyclophosphamide, and Dexamethasone; TD: Thalidomide and Dexamethasone, TAD: Thalidomide, Adriamycin, and Dexamethasone, MPT: Melphalan, Prednisone, and Thalidomide, T-DECP: Thalidomide, Cisplatin, Etoposide, Cyclophosphamide, and Dexamethasone; CTP: Cyclophosphamide, Thalidomide, and Dexamethasone, and PR: partial response.

small (41 patients). Thus the data of these patients were not included in this study. Compared to patients in thalidomidebased group, patients in bortezomib-based group had more elder patients $(p=0.0001)$, higher BM plasma cell percentage $(p=0.012)$, and better response rate $(p<0.0001)$.

3.2. Patient Outcome in the Entire Cohort. The median estimated follow-up for the cohort was 24.3 months with $72.1 \%$ alive at last follow-up. The median OS was 55.8 months and PFS was 28.0 months for the entire patients. In this study, it showed a significant better survival in patients who at least achieve PR in the first-line therapy than those who did not, median OS was 63.4 versus 53.7 months, and 5-year survival was $50.9 \%$ versus $38.3 \%(p<0.0001)$ (Figure 1 ).

3.3. Validate Prognostic Value of ISS System in the Entire Cohort. The prognostic value of ISS system was evaluated in the total population of 1,016 patients. Patients were divided into stages I/II/III according to ISS system, and corresponding proportion in each stage was $22.7 \% / 33.6 \% / 43.7 \%$, respectively. The median OS for ISS stages I/II/III was not reached/55.4 months/41.7 months (Figure 2), and the median PFS was $30 / 29.5 / 25$ months $(p=0.072)$, respectively. From these data, we can conclude that ISS system can predict prognosis for OS, but for PFS in MM patients in the entire cohort.

3.4. Validate Prognostic Value of ISS System in BortezomibBased or Thalidomide-Based Group. In order to discern the impact of novel agents to the ISS system, subgroup analyses for OS were also performed in patients who received bortezomib-based treatment and thalidomide-based treatment in the first-line therapy.

In bortezomib-based group, 170 patients were in ISSI, 236 in ISS-II, and 303 in ISS-III, with median OS being not reached/57.5 months/42.0 months and 3-year survival was $77.7 \% / 75.8 \% / 53.4 \%$, respectively (Figure 3). Statistical difference was verified between ISS-I and ISS-III $(p<$ $0.0001)$, ISS-II and ISS-III $(p<0.0001)$, and ISS-I and 


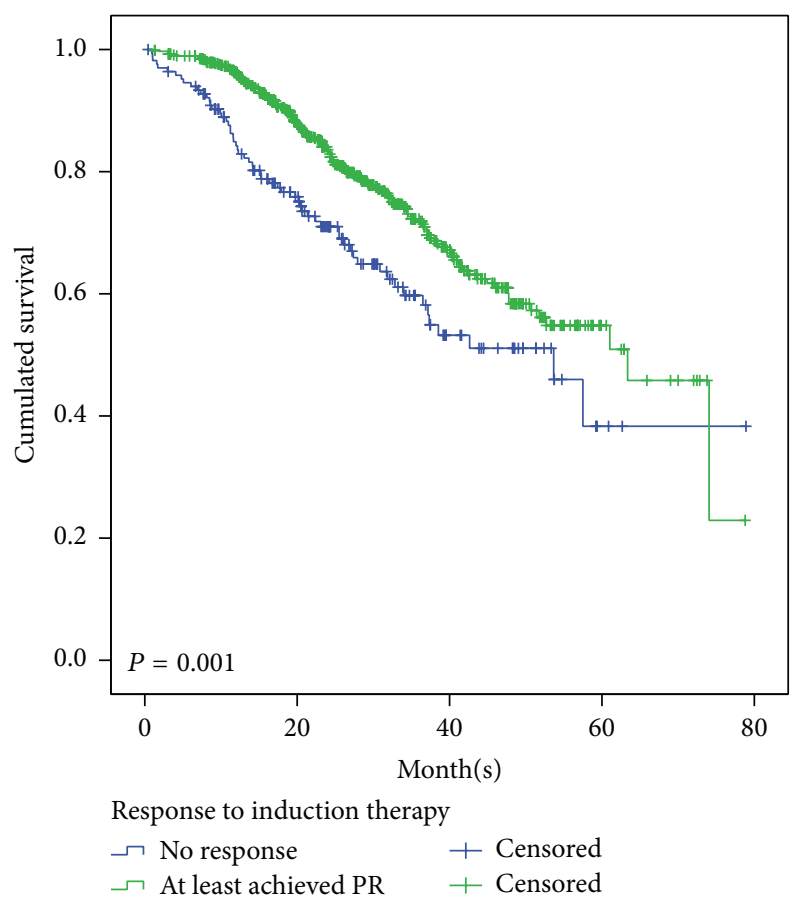

FIgURE 1: Overall survival (OS) for 1016 patients according to the response to the first-line therapy who at least achieved PR and who were below PR.

ISS-II ( $p=0.038)$. In order to validate ISS system in transplant and nontransplant patients, we further stratified bortezomib-based patients into two subgroups according to whether they underwent transplantation after induction therapy. In bortezomib-based group, 177 patients underwent transplantation and 532 patients did not, the median OS was 51.8 months versus 57.5 months, respectively. The 3 -year survival was $76.1 \%$ versus $69.2 \%$ versus $56.2 \%$ in nontransplantation group and $89.7 \%$ versus $86.7 \%$ versus $42.3 \%$ in transplantation group, respectively. The similar result was showed in transplantation and nontransplantation group, and no statistical difference was showed between ISS-I and ISSII in bortezomib-based transplantation $(p=0.413)$ and nontransplantation groups ( $p=0.056$ ).

In thalidomide-based group, 61 patients were classified in ISS-I, 105 in ISS-II, and 141 in ISS-III, with median OS being not reached, 55.4 months, and 41.7 months, respectively (Figure 4). Statistically significant difference in survival was confirmed between three stages, ISS-I and ISS-II $(p=0.024)$, ISS-I and ISS-III $(p<0.0001)$, and ISS-II and ISS-III ( $p=0.047)$, respectively. Among these patients, 282 patients underwent transplantation and 25 patients did not. Because the number in transplantation group is quite small, we do not carry out analysis in transplant group. In nontransplant group, 54 patients were in ISS-I, 95 in ISS-II, and 133 in ISSIII. There was statistical difference between ISS-I and ISSII $(p=0.009)$ and ISS-I and ISS-III $(p<0.0001)$, but no statistical difference between ISS-II and ISS-III $(p=0.089)$. Although ISS prognostic significance disappeared between ISS-II and ISS-III in thalidomide-based nontransplant group,

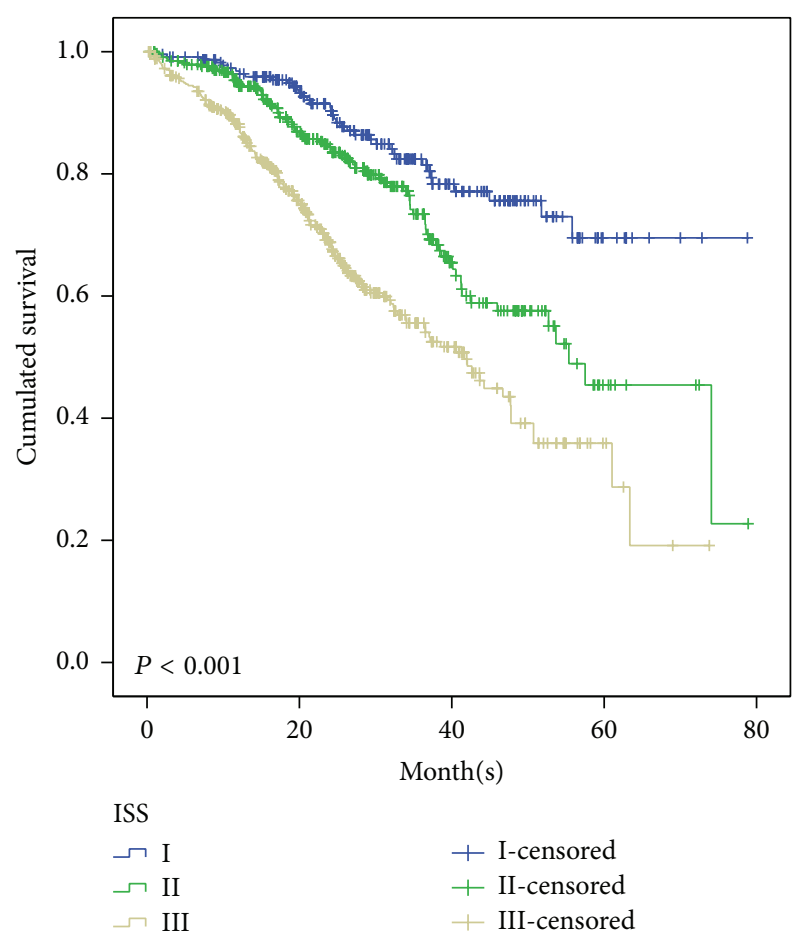

FIGURE 2: Overall survival for 1016 patients with newly diagnosed symptomatic multiple myeloma according to the ISS stages I, II, and III, who were treated with novel agents as the first-line strategy.

this may be due to the small number in ISS stages II and III; more studies are needed in the future.

\section{Discussion}

Multiple myeloma (MM) is characterized by heterogeneity in the clinical course and risk stratification is vital for prediction of prognosis. ISS is the most important prognostic system for MM in the past ten years. This system predicts survival of newly diagnosed MM patients by using two routine and inexpensive pieces of laboratory data and separated patients into three stages with a distinct prognosis [4]. Although ISS system was wildly used in Chinese myeloma patients in the past decade, the original analysis of ISS system from Greipp et al. did not include Chinese patients' data. Besides these, the survival of MM has dramatically changed by the introduction of novel agents, and nowadays the majority of patients received novel agent based treatment in the first-line therapy. Thus, the initial question that motivates our study was to determine whether ISS is suitable in the era of novel agents and in Chinese MM patients. This study aimed to provide outcome data for patients actually exposed to novel agents at first-line treatment.

In this analysis, we enrolled consecutive patients; thus the results may be more appropriate to the general group of myeloma patients, for patients enrolled in clinical trials were selected by some screening conditions. In this study, all of patients exposed to novel agents in first-line therapy. Survival in patients who achieved at least PR at induction 


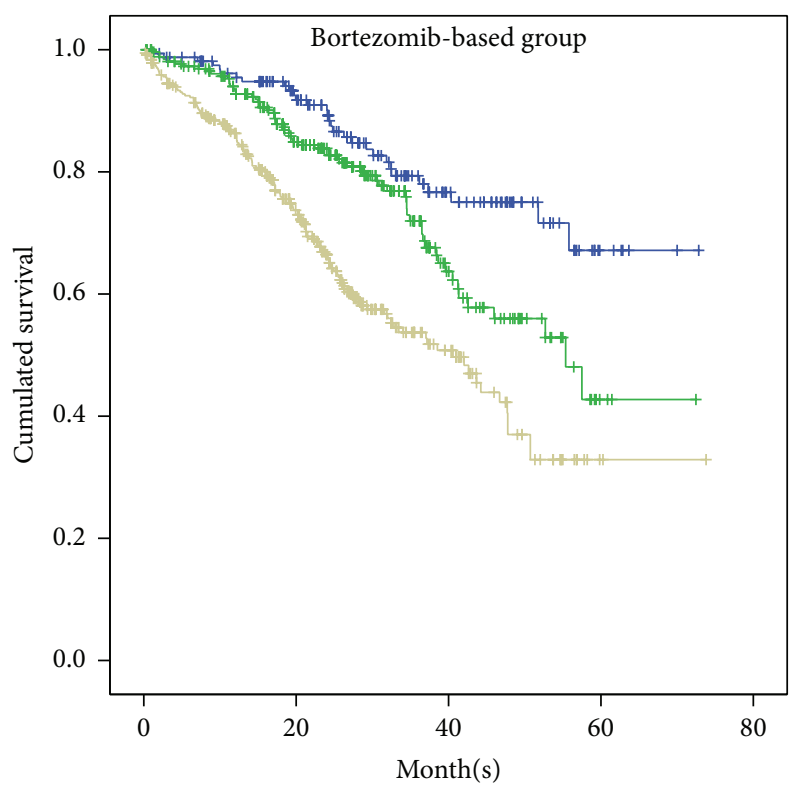

ISS

$$
\begin{array}{ll}
\neg \text { I } & + \text { I-censored } \\
\neg \text { II } & + \text { II-censored } \\
\neg \text { III } & + \text { III-censored }
\end{array}
$$

Figure 3: Overall survival for 709 patients with newly diagnosed symptomatic multiple myeloma according to the ISS stages I, II, and III, who were treated with bortezomib-based treatment as first-line therapy.

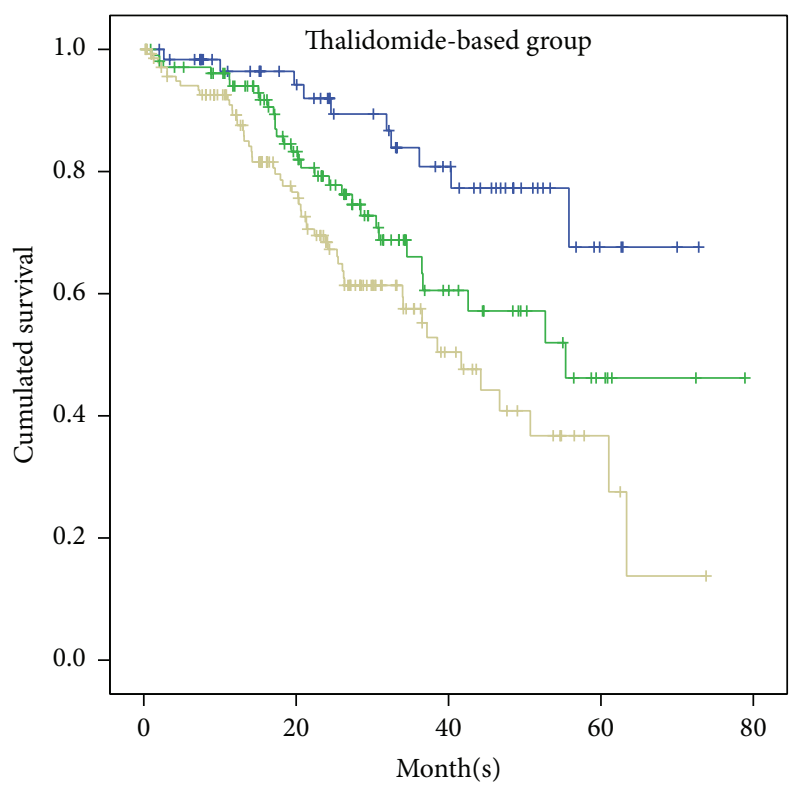

ISS

$$
\begin{array}{ll}
\neg \text { I } & + \text { I-censored } \\
\neg \text { II } & + \text { II-censored } \\
\neg \text { III } & + \text { III-censored }
\end{array}
$$

FIGURE 4: Overall survival for 307 patients with newly diagnosed symptomatic multiple myeloma according to the ISS stages I, II, and III, who were treated with thalidomide-based treatment as first-line therapy. therapy is much better than those who did not responsd, due to better outcome with more aggressive therapy. These results confirmed the Dimopoulos et al.s observations [12].

The ISS system still has prognostic significance value when applied to the total 1016 patients, with median OS for ISS stages I/II/III being not reached/55.4 months/ 41.7 months $(p<0.001)$. When ISS was proposed the median OS of MM patients in ISS stages I/II/III was 64, 44, and 29 months, respectively. Thus this study indicates that the survival of patients in each ISS stage is significantly improved in the era of novel agents. This significance partly disappeared when ISS implied to patients who received bortezomib-based regimens in the first-line treatment. We demonstrated the ISS system still has prognostic value in the era of novel agents in Chinese patients with MM, while in subgroup analysis it is not fully applicable and limited prognostic value in patients receiving bortezomib-based treatment in first-line therapy.

There is few data on the applicability of ISS in bortezomibbased treatment in the first-line therapy in literature. From previous studies we can indicate that MM patients can achieve deeper response by the use of novel agents, improved PFS and OS [13]. A meta-analysis performed by Zou et al. showed the addition of bortezomib to first-line therapy did significantly prolong OS compared with conventional therapy alone [14]. Some studies have showed that bortezomib-based regimens can improve outcome of patients with $t(4 ; 14)$, deletion of chromosome 13 , and deletion of $17 \mathrm{p}$, respectively [15-17]. This study also figured out patients with either $t(4 ; 14)$ or del(17p) presented in a higher ISS stage. We can find out that bortezomib has shown survival benefit in myeloma and overcome specific cytogenetic risk features in MM patients. This may partly explain in our study no statistical difference in OS between ISS stages I and II.

The ISS system was used as an independent prognostic system in the past, but it was unable to reflect the cytogenetic abnormalities of MM. Some new prognostic factors were increasingly found, such as fluorescent in situ hybridization (FISH), karyotype, and serum-free light chain [18-21]. These new prognostic factors can overcome this deficiency and provide cytogenetic or molecular genetics-based risk classification for MM patients. Many efforts have been made, such as proposing a new stage system by combination of ISS with FISH [22]. A recent study from IMWG combined ISS, $\mathrm{CA}$, and LDH data to define Revised International Staging System (R-ISS) by following three risk categories: R-ISS I including ISS stage I, no high-risk CA [del(17p) and/or t $(4 ; 14)$ and/or t(14;16)], and normal LDH level; R-ISS III including ISS stage III and high-risk CA or high LDH level; and RISS II including all the other combinations. The data of RISS were enrolled on 11 clinical trials from 2005 to 2013. All patients received new drugs based chemotherapy as upfront treatment. The 5-year OS rate in R-ISS I, II, and III was $82 \%, 62 \%$, and $40 \%$, respectively. In our data, few patients had the data of chromosomal abnormalities (CA) detected by interphase fluorescent in situ hybridization after CD138 plasma cell purification. Compared with these IMWG studies, the majority of patients were in intermediate-risk group; in our study, $43.7 \%$ of patients were in high-risk group (ISS stage III). This distribution may explain worse survival in 
our study in three stage groups. The R-ISS system can predict prognosis on OS in patients who did receive proteasome inhibitor based treatment, while in our study the ISS system cannot clearly distribute the OS of MM patients in ISS stages I and II. One interpretation might be that, compared with RISS system, ISS system may wrongly allocate a certain group of patients with poor prognosis in lower ISS stage [23].

There are many restrictive conditions for these new variables, for example, no consensus in standard classifications, not being easily available, and being too expensive. Their applications were limited by these passive factors. Thus, although novel prognostic factors such as FISH, karyotype, and serum-free light chain are important in MM risk stratification, the prognostic value of traditional serum markers still deserves attention. It can be an important component of new staging system in the future. Reevaluating the prognostic value of ISS system now is beneficial for the future research for a new staging system.

Because the initial retrospective study design from which these data are obtained was focused on clinical features and outcome in Chinese patients, the data analysis was presented with a number of challenges including (1) inconsistencies in patients feature among two groups, (2) the inability to study the effect of novel agents by using the same regimens in each group, and (3) needing a very long follow-up time and a very large patients' series to prove OS benefit in the era of new agents. Unfortunately, we could not evaluate the impact of adverse genetic markers in our cohort of patients because FISH studies were performed in a minority of patients.

\section{Conclusion}

In conclusion, our data is the first multicenter retrospective study in Chinese myeloma patients that validates ISS value in a large number of unselected patients. The results demonstrated that International Staging System still has prognostic value in the era of novel agents in Chinese patients with MM. However, that ISS system is not fully applicable in patients receiving bortezomib-based therapy at first-line treatment. These findings suggest that ISS system is predictive for OS of Chinese MM patients in the era of novel agents, but value is limited in PFS and in patients who were exposed to bortezomib in the first-line therapy. Bortezomib may have the potential to partially overcome adverse effect of risk factors on survival, especially in higher stage of ISS system. Further study is needed to develop more suitable staging system applied to MM patients in the era of novel agents which can reflect not only tumor burden and patient's condition, but also genetic risk classification.

\section{Conflict of Interests}

The authors declare that they have no competing interests.

\section{Authors' Contribution}

Jing Lu, Jin Lu, and Aijun Liu are equal contributors and are co-first authors.

\section{Acknowledgments}

This study was granted by Natural Science Foundation of China (Grant nos. 81172248 and 81470360) and Tian Jin Municipal Special Foundation for Anticancer (Grant no. 12ZCDZSY17600).

\section{References}

[1] S. V. Rajkumar, "Treatment of multiple myeloma," Nature Reviews Clinical Oncology, vol. 8, no. 8, pp. 479-491, 2011.

[2] R. A. Kyle and S. V. Rajkumar, "Criteria for diagnosis, staging, risk stratification and response assessment of multiple myeloma," Leukemia, vol. 23, no. 1, pp. 3-9, 2009.

[3] B. G. Durie and S. E. Salmon, "A clinical staging system for multiple myeloma correlation of measured myeloma cell mass with presenting," Cancer, vol. 36, no. 3, pp. 842-854, 1975.

[4] P. R. Greipp, J. S. Miguel, B. G. M. Dune et al., "International staging system for multiple myeloma," Journal of Clinical Oncology, vol. 23, no. 15, pp. 3412-3420, 2005.

[5] I. Turesson, R. Velez, S. Y. Kristinsson, and O. Landgren, "Patterns of improved survival in patients with multiple myeloma in the twenty-first century: a population-based study, Journal of Clinical Oncology, vol. 28, no. 5, pp. 830-834, 2010.

[6] E. Kastritis, K. Zervas, A. Symeonidis et al., "Improved survival of patients with multiple myeloma after the introduction of novel agents and the applicability of the International Staging System (ISS): an analysis of the Greek Myeloma Study Group (GMSG)," Leukemia, vol. 23, no. 6, pp. 1152-1157, 2009.

[7] M. A. Dimopoulos, E. Kastritis, E. Michalis et al., "The international scoring system (ISS) for multiple myeloma remains a robust prognostic tool independently of patients' renal function," Annals of Oncology, vol. 23, no. 3, pp. 722-729, 2012.

[8] S.-H. Yang, H.-W. Teng, Y.-C. Hong et al., "International Staging System predicts prognosis of Chinese patients with multiple myeloma across different calendar periods with application of novel agents," Annals of Hematology, vol. 91, no. 1, pp. 93-102, 2012.

[9] K. C. Anderson, M. Alsina, W. Bensinger et al., "Multiple myeloma, version 1.2013: featured updates to the NCCN guidelines," Journal of the National Comprehensive Cancer Network, vol. 11, no. 1, pp. 11-17, 2013.

[10] P. Moreau, J. San Miguel, H. Ludwig et al., "Multiple myeloma: ESMO clinical practice guidelines for diagnosis, treatment and follow-up," Annals of Oncology, vol. 24, no. 6, Article ID mdt297, pp. 133-137, 2013.

[11] B. G. M. Durie, J.-L. Harousseau, J. S. Miguel et al., "International uniform response criteria for multiple myeloma," Leukemia, vol. 20, no. 9, pp. 1467-1473, 2006.

[12] M. A. Dimopoulos, E. Kastritis, S. Delimpasi et al., "Multiple myeloma in octogenarians: clinical features and outcome in the novel agent era," European Journal of Haematology, vol. 89, no. 1, pp. 10-15, 2012.

[13] F. Gay, A. Larocca, P. Wijermans et al., "Complete response correlates with long-term progression-free and overall survival in elderly myeloma treated with novel agents: analysis of 1175 patients," Blood, vol. 117, no. 11, pp. 3025-3031, 2011.

[14] Y. Zou, M. Lin, Z. Sheng, and S. Niu, "Bortezomib and lenalidomide as front-line therapy for multiple myeloma," Leukemia and Lymphoma, vol. 55, no. 9, pp. 2024-2031, 2014. 
[15] H. Avet-Loiseau, X. Leleu, M. Roussel et al., "Bortezomib plus dexamethasone induction improves outcome of patients with $\mathrm{t}(4 ; 14)$ myeloma but not outcome of patients with del(17p)," Journal of Clinical Oncology, vol. 28, no. 30, pp. 4630-4634, 2010.

[16] S. Jagannath, P. G. Richardson, P. Sonneveld et al., "Bortezomib appears to overcome the poor prognosis conferred by chromosome 13 deletion in phase 2 and 3 trials," Leukemia, vol. 21, no. 1, pp. 151-157, 2007.

[17] K. Neben, H. M. Lokhorst, A. Jauch et al., "Administration of bortezomib before and after autologous stem cell transplantation improves outcome in multiple myeloma patients with deletion 17p," Blood, vol. 119, no. 4, pp. 940-948, 2012.

[18] J. R. Mikhael, D. Dingli, and V. Roy, "Management of newly diagnosed symptomatic multiple myeloma: updated Mayo Stratification of Myeloma and Risk-Adapted Therapy (mSMART) consensus guidelines," Mayo Clinic Proceedings, vol. 88, no. 4, pp. 360-376, 2013.

[19] A. Dispenzieri, R. Kyle, G. Merlini et al., "International Myeloma Working Group guidelines for serum-free light chain analysis in multiple myeloma and related disorders," Leukemia, vol. 23, no. 2, pp. 215-224, 2009.

[20] R. Fonseca, B. Barlogie, R. Bataille et al., "Genetics and cytogenetics of multiple myeloma: a workshop report," Cancer Research, vol. 64, no. 4, pp. 1546-1558, 2004.

[21] A. Corso, M. Galli, S. Mangiacavalli et al., "Response-adjusted ISS (RaISS) is a simple and reliable prognostic scoring system for predicting progression-free survival in transplanted patients with multiple myeloma," American Journal of Hematology, vol. 87, no. 2, pp. 150-154, 2012.

[22] H. Avet-Loiseau, B. G. M. Durie, M. Cavo et al., "Combining fluorescent in situ hybridization data with ISS staging improves risk assessment in myeloma: an International Myeloma Working Group collaborative project," Leukemia, vol. 27, no. 3, pp. 711-717, 2013.

[23] A. Palumbo, H. Avet-Loiseau, S. Oliva et al., "Revised international staging system for multiple myeloma: a report from International Myeloma Working Group," Journal of Clinical Oncology, 2015. 


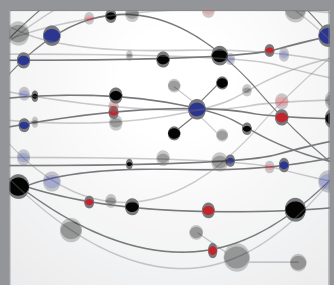

The Scientific World Journal
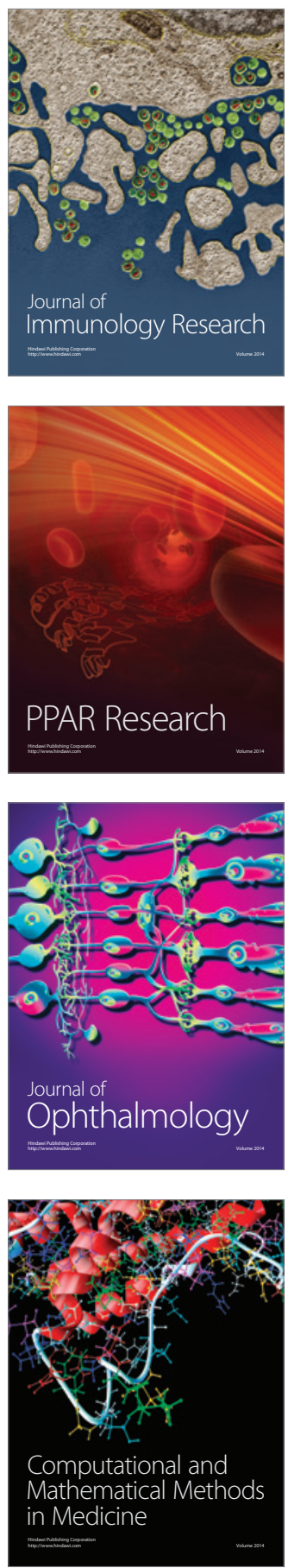

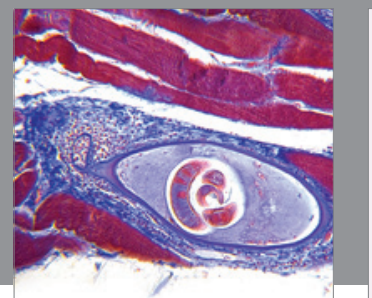

Gastroenterology

Research and Practice
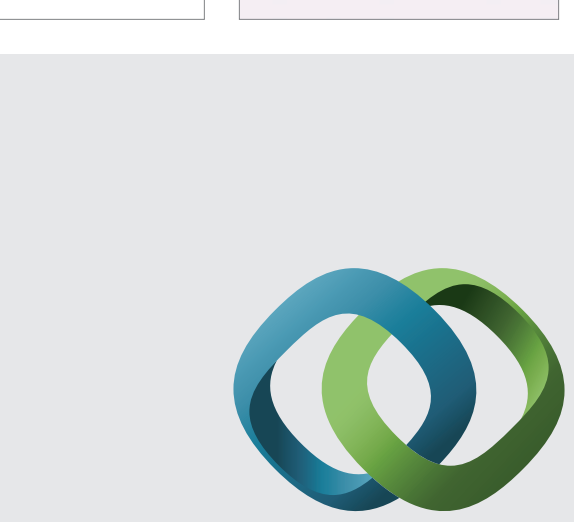

\section{Hindawi}

Submit your manuscripts at

http://www.hindawi.com
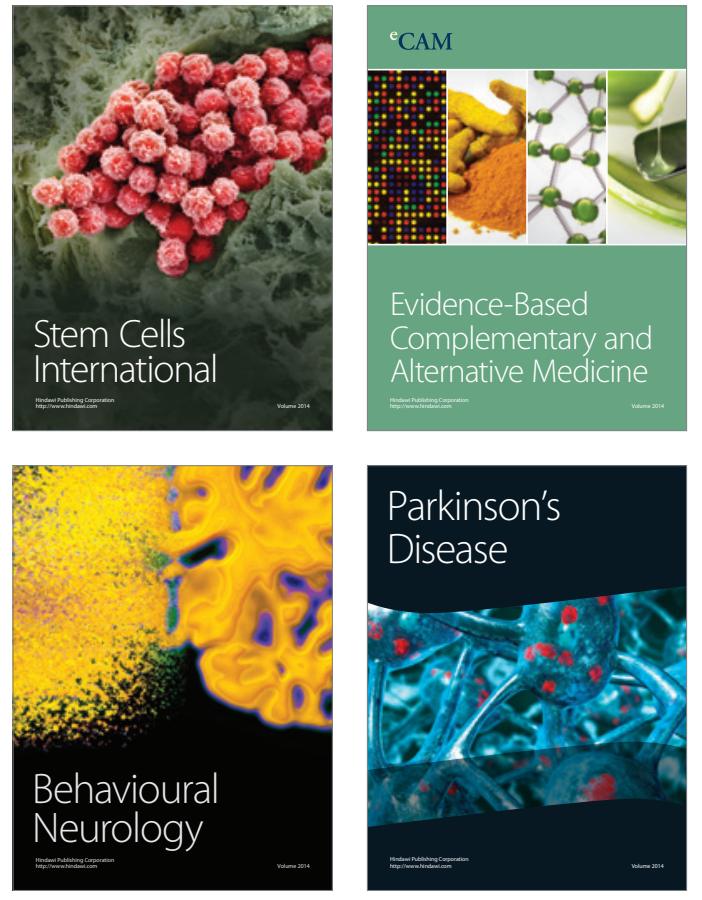
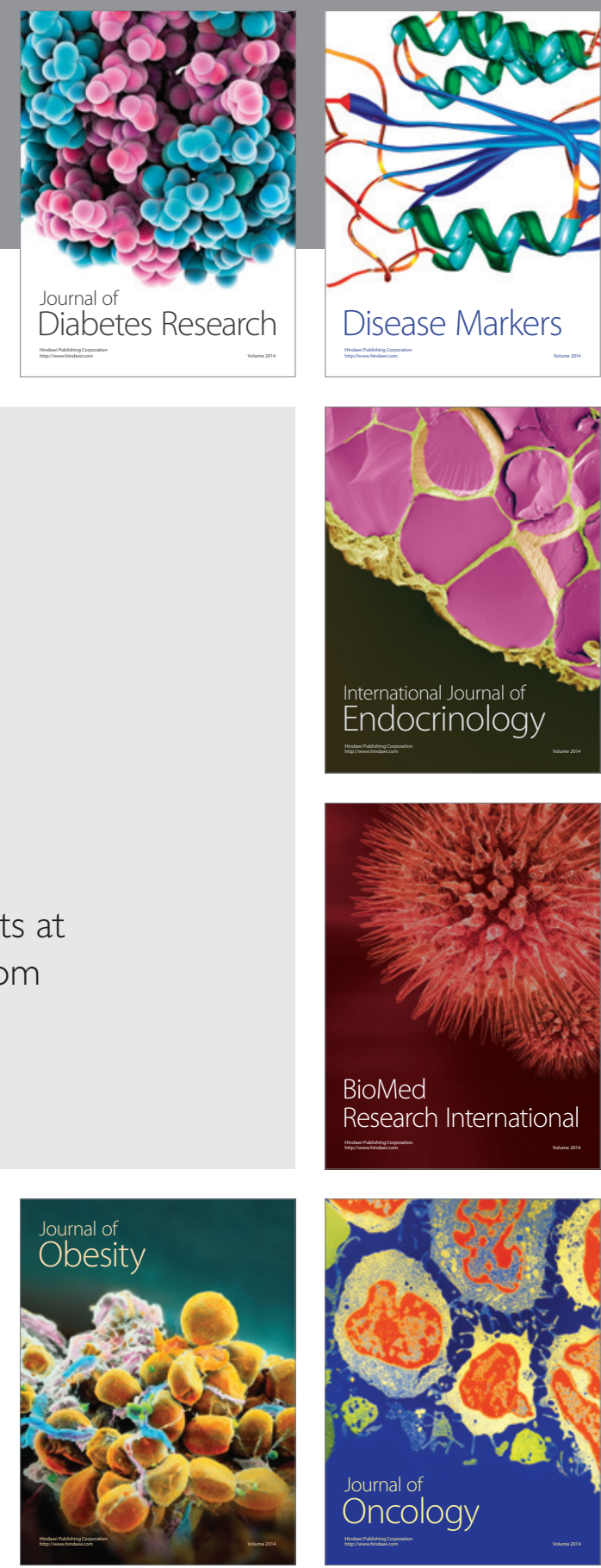

Disease Markers
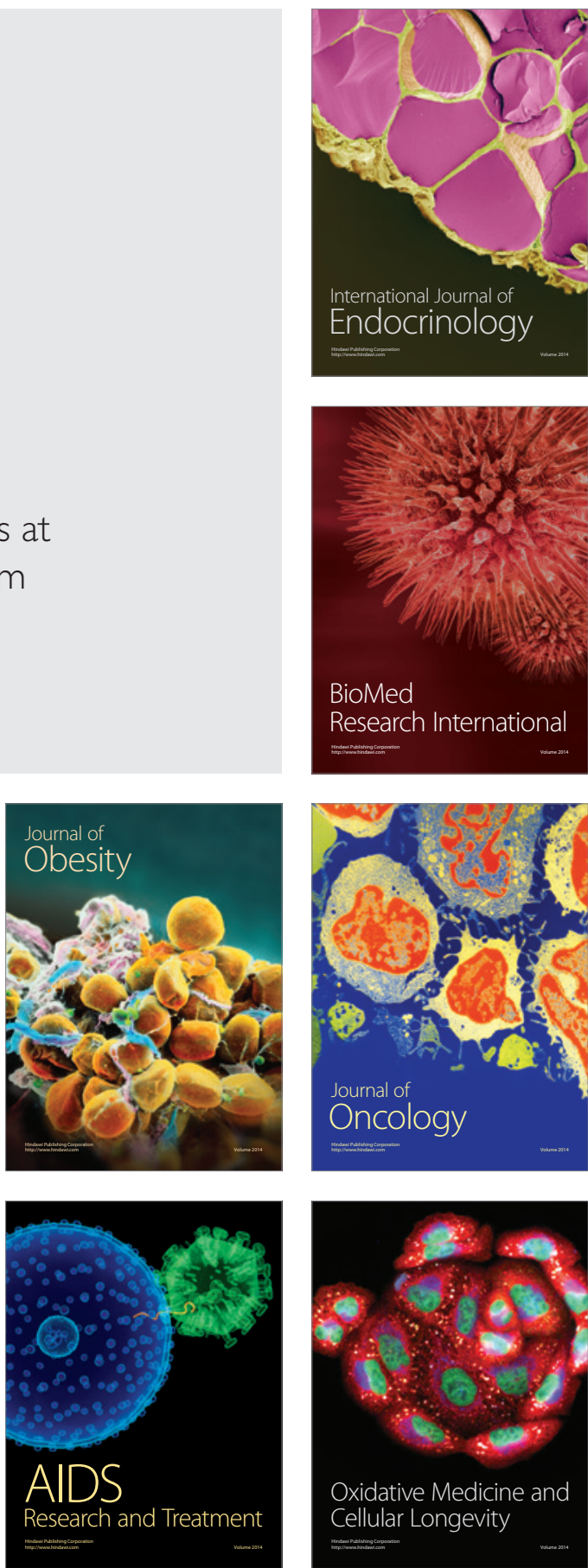